\title{
ESTUDO COMPARATIVO ENTRE AS TENÓLISES CONVENCIONAIS E COM DESPERTAR INTRAOPERATÓRIO REALIZADAS NA REGIÃO DO TÚNEL OSTEOFIBROSO FLEXOR DAS MÃOS (ZONA 2)
}

\author{
COMPARATIVE STUDY BETWEEN TRADITIONAL TENOLYSES AND WITH \\ INTRAOPERATIVE AWAKENING PERFORMED ON THE FLEXOR \\ OSTEOFIBROUS TUNNEL REGION OF THE HANDS (ZONE 2)
}

Tiago Guedes da Motta Mattar ${ }^{1}$, Rames Mattar Junior ${ }^{2}$, Alvaro Baik Cho ${ }^{3}$, Emygdio Jose Leomil de Paula Marcelo Rosa Rezende 4

\section{RESUMO}

Objetivo: As tenólises dos tendões flexores na zona 2 são procedimentos difíceis e um verdadeiro desafio na cirurgia da mão. Com o objetivo de comparar os resultados obtidos entre as tenólises com despertar intraoperatório, realizadas sob anestesia locorregional (grupo 1), daqueles obtidos com as tenólises convencionais, realizadas sob anestesia geral ou bloqueio total do plexo braquial (grupo 2), os autores realizam um estudo prospectivo e controlado. Métodos: Foram avaliados 22 pacientes com 39 dedos portadores de lesão de tendão flexor na zona 2 que evoluíram para aderências. Todos os pacientes foram operados após três meses e antes de um ano da sutura tendinosa primária. Todos apresentavam limitação da movimentação ativa sem melhora com a reabilitação. Os grupos 1 e 2 mostraram-se homogêneos em relação à idade dos pacientes, sexo, comprometimento pré-operatório e ausência de lesões ou patologias associadas. Todos os pacientes foram avaliados de acordo com a movimentação ativa (TAMs) no período pré-operatório e com seis meses de pós-operatório. Resultados: A análise estatística dos dados obtidos nos grupos 1 e 2 demonstra que as tenólises realizadas pelas duas técnicas proporcionam bons resultados. Ao comparar os resultados da movimentação ativa total após seis meses da tenólise, observa-se que os pacientes do grupo 1, tratados pela técnica do despertar intraoperatório, apresentavam melhores resultados. Conclusões: As tenólises dos flexores na zona 2 proporcionam bons resultados em termos de ganho de amplitude articular. A técnica de despertar intraoperatório com anestesia locorregional proporciona resultados mais satisfatórios que a técnica convencional.

Descritores - Traumatismos dos dedos; Traumatismos dos tendões; Tendões/cirurgia; Reabilitação

\section{ABSTRACT}

Objective: Flexor tendon tenolysis on zone 2 is a difficult and really challenging for hand surgery. With the objective of comparing the results obtained between tenolyses with intraoperative awakening, performed with locoregional anesthesia (group 1), from those obtained with traditional tenolysis performed under general anesthesia or total blockage of the brachial plexus (group 2), the authors conducted a prospective and controlled study. Methods: 22 patients with 39 fingers with flexor tendon injuries on zone 2 evolving to adherences were assessed. All patients were operated after three months and before one year of the primary tendinous suture. All patients showed limited active motion not improved by rehabilitation. Groups 1 and 2 showed to be homogenous concerning patients' age and gender, preoperative compromising, and absence of associated injuries or pathologies. All patients were assessed according to active motion (TAMs) both preoperatively and at 6 months postoperatively. Results: The statistical analysis of data obtained for groups 1 and 2 shows that the tenolysis performed with both techniques produce good results. By comparing the results for total active motion after six months of the tenolysis, group 1 patients (treated by the intraoperative awakening technique) were found to present better outcomes. Conclusions: Flexor tenolysis on zone 2 provide good results in terms of joint range of motion. The intraoperative awakening technique with locoregional anesthesia provides improved outcomes when compared to the traditional technique.

Keywords - Finger injuries; Tendon injuries; Tendons/surgery; Rehabilitation

1 - Residente do $3^{\circ}$. ano da Disciplina de Ortopedia da Faculdade de Medicina do ABC.

2 - Professor Associado do Departamento de Ortopedia e Traumatologia da FMUSP.

3 - Médico Assistente da Disciplina de Ortopedia da Faculdade de Medicina do ABC e Doutor pelo Departamento de Ortopedia e Traumatologia da FMUSP.

4 - Médico Assistente Doutor do Departamento de Ortopedia e Traumatologia da FMUSP.

Trabalho realizado nos Hospitais Albert Einstein, Professor Edmundo Vasconcelos, Faculdade de Medicina do ABC e Instituto de Ortopedia e Traumatologia do HC/FMUSP. Correspondência: Rames Mattar Junior, Av. Jurema, 534, apt 251 - 04079-001 - São Paulo, SP. Tel.: (11) 5051-7777. E-mail: rames@usp.br 


\section{INTRODUÇÃO}

As lesões dos tendões flexores na zona 2 são graves, pois afetam a função nobre de preensão da mão e seu tratamento é complexo. Sua reconstrução é difícil porque a sutura exige resistência para suportar a tração dos músculos flexores e, ao mesmo tempo, necessidade de manter a capacidade de deslizamento para promover a excursão necessária para o movimento dos dedos. Além disso, na zona 2, os tendões flexores apresentam-se, quase na totalidade, envoltos por uma bainha sinovial e recebem nutrição sanguínea pelas vínculas. Os tendões superficial e profundo dos dedos apresentam complexa relação de deslizamento e excursão e agem em várias articulações. Tais características anatômicas tornam seu reparo cirúrgico mais difícil ${ }^{(1)}$.

A cicatrização dos tendões tem sido motivo de controvérsia por muitos anos. Inicialmente, acreditava-se que os tendões cicatrizassem através de fibroblastos da bainha digital ou de tecidos vizinhos, e que a nutrição seria proporcionada através de aderências nessas estruturas $^{(2,3)}$. Entretanto, demonstrou-se que os tendões podem cicatrizar satisfatoriamente através da nutrição sinovial exclusivamente e o colágeno necessário para cicatrização pode ser produzido pelos tenócitos ${ }^{(4-6)}$.

A cicatrização intrínseca dos tendões inicia-se por proliferação de células do epitendão que crescem ao longo do tendão e ao nível da lesão, formando uma espécie de "calo", de forma similar ao que ocorre na pele ou no tecido ósseo ${ }^{(4,7)}$. Tardiamente, fibroblastos e tenócitos invadem o "calo" e produzem colágeno que irá se organizar e alinhar produzindo um tendão normal. Parece que o suporte dado pela nutrição sinovial é suficiente para manter todo esse processo. As aderências formadas na cicatrização extrínseca parecem não ser fundamentais para a cicatrização ou nutrição do tendão ${ }^{(7)}$.

A função básica dos tendões flexores é o deslizamento que promove a movimentação do dedo. As aderências tendinosas podem ocorrer devido aos diversos mecanismos de lesão dos tendões (ferimentos cortocontusos, laceração, avulsão ou esmagamento), manipulação cirúrgica, como consequência de fraturas ou processos infecciosos ${ }^{(8,9)}$. Algumas aderências podem ser tratadas com sucesso com técnicas apropriadas de reabilitação. Quando não há melhora e existe grande diferença entre a amplitude de movimentação passiva e ativa, poderá haver indicação para o procedimento de tenólise ${ }^{(9,10)}$. A tenólise consiste na liberação de todas as aderências entre o tendão flexor e as estruturas vizinhas, preservando a anatomia local, principalmente o sistema de polias, cápsula articular, placa volar, vasos e nervos ${ }^{(9-12)}$.
A maioria dos autores recomenda que a tenólise não deva ser realizada antes dos três meses e depois de um ano da cirurgia de tenorrafia primária. Antes dos três meses o processo de cicatrização do tendão poderá não proporcionar resistência mecânica suficiente para suportar a agressão cirúrgica da tenólise. Por outro lado, a cirurgia realizada após um ano, usualmente, é tecnicamente mais difícil em virtude da magnitude das aderências e rigidez $\operatorname{articular}^{(13-17)}$.

Uma vez realizada a liberação cirúrgica, o arco de movimento do dedo comprometido deve ser restabelecido e há necessidade de iniciar precocemente um programa de reabilitação que inclui, principalmente, a movimentação ativa e passiva do dedo operado. A reabilitação deve ser iniciada logo após o procedimento cirúrgico e continuar até que a amplitude de movimentação se mantenha estável ${ }^{(12,17)}$.

Há controvérsia em relação ao tipo de anestesia e técnica cirúrgica a ser adotada. Enquanto alguns autores utilizam a anestesia geral ou bloqueio do plexo braquial, outros preferem bloqueios mais distais, com o objetivo de manter a função da musculatura flexora (extrínseca ou extrínseca e intrínseca) e permitir o teste da liberação dos flexores aderidos pela movimentação ativa após o despertar intraoperatório. Schneider e Hunter recomendam o uso de anestésicos locais associados à sedação endovenosa para que o paciente possa participar ativamente do procedimento de liberação dos tendões flexores ${ }^{(9,18)}$. Outros autores recomendam o uso de anestesia geral, principalmente quando há ferimentos mais extensos ou quando o tempo de uso do manguito pneumático excede em uma hora ${ }^{(10)}$. Há, ainda, o argumento de que alguns pacientes não aceitam ou não reagem bem ao despertar intraoperatório. Quando a tenólise é realizada sob anestesia geral ou bloqueio do plexo braquial, o cirurgião deverá estar convicto de que todas as aderências foram liberadas e que o paciente irá conquistar toda a amplitude de movimentação possível. Alguns autores recomendam a realização de uma incisão adicional no punho para permitir o acesso e a tração do tendão flexor liberado, com os objetivos de testar o grau de amplitude de movimento conseguido e liberar aderências residuais ${ }^{(10)}$.

$\mathrm{Na}$ maioria das vezes, utiliza-se a mesma incisão adotada na cirurgia de tenorrafia primária, seja ela médio-lateral ou do tipo Brunner. O tendão aderido é usualmente liberado por técnicas delicadas de dissecção que preservam as estruturas vizinhas. Quando se utiliza a técnica do despertar intraoperatório com aneste- 
sia locorregional, a liberação é realizada quase que por etapas. Após dissecção e liberação do tendão na área considerada crítica, o paciente é acordado pela ação da droga flumazenil, sendo, então, solicitado que realize uma flexão ativa forçada. Caso essa liberação não seja suficiente para proporcionar todo o ganho de amplitude de movimento possível (movimentação ativa igual à passiva), o procedimento de tenólise é estendido para área mais proximal ou distal.

Não existem estudos comparativos entre a técnica convencional, realizada sob anestesia geral ou bloqueio total do plexo braquial, e a com despertar intraoperatório sob anestesia locorregional distal.

Com o objetivo de comparar essas duas técnicas, realizamos um estudo prospectivo controlado em pacientes submetidos à tenólise para tratamento de aderências tendinosas na zona 2 do túnel osteofibroso flexor.

\section{MÉTODOS}

Entre 2000 e 2008 foram operados 12 pacientes com 20 dedos portadores de aderências tendinosas no sistema flexor na região do túnel osteofibroso (zona 2) com a técnica do despertar intraoperatório utilizando anestesia locorregional, que chamaremos de grupo 1. O grupo controle (grupo 2) foi constituído por dez pacientes com 19 dedos portadores de aderências tendinosas na zona 2 e que foram submetidos a tenólises convencionais sob anestesia geral ou bloqueio total do plexo braquial.

Todos os pacientes apresentavam aderências tendinosas na zona 2 com mais de três meses e menos de um ano da tenorrafia. Foram excluídos pacientes que apresentavam outras lesões na mão, como fraturas, lesão de nervos periféricos, perda do revestimento cutâneo, etc. Não foram incluídos neste estudo pacientes com diabetes, vasculopatias, artrites, artrose e outras patologias que pudessem comprometer o resultado funcional do procedimento de tenólise e reabilitação. Todos os pacientes foram operados pela mesma equipe cirúrgica e todos foram avaliados no pré-operatório e aos seis meses de pós-operatório obedecendo ao mesmo protocolo.

Os dados dos pacientes relacionados com idade, sexo e dedos comprometidos encontram-se na tabela 1 .

A técnica cirúrgica de ambos os grupos baseou-se na realização da mesma incisão utilizada para a tenorrafia (tipo Brunner ou médio-lateral), dissecção dos tendões flexores mantendo o sistema de polias, liberação de todas as aderências e mobilização do dedo.

No grupo 1 o paciente foi submetido a bloqueio anestésico com bupivacaína a $0,5 \%$ na região do punho e
Tabela 1 - Distribuição do dados relacionados com sexo e idade dos pacientes estudados

\begin{tabular}{|c|c|c|c|c|c|c|}
\hline \multirow[t]{2}{*}{ Dedo } & \multicolumn{3}{|c|}{ Grupo 1} & \multicolumn{3}{|c|}{ Grupo 2} \\
\hline & & Idade & Sexo & & Idade & Sexo \\
\hline 1 & caso 1 dedo 1 & 22 & masculino & caso 1 dedo 1 & 33 & masculino \\
\hline 2 & caso 1 dedo 2 & & & caso 1 dedo 2 & & \\
\hline 3 & caso 2 dedo 1 & 37 & masculino & caso 1 dedo 3 & & \\
\hline 4 & caso 2 dedo 2 & & & caso 2 dedo 1 & 24 & feminino \\
\hline 5 & caso 3 dedo 1 & 32 & feminino & caso 3 dedo 1 & 55 & masculino \\
\hline 6 & caso 4 dedo 1 & 42 & masculino & caso 3 dedo 2 & & \\
\hline 7 & caso 4 dedo 2 & & & caso 3 dedo 3 & & \\
\hline 8 & caso 4 dedo 3 & & & caso 4 dedo 1 & 25 & masculino \\
\hline 9 & caso 5 dedo 1 & 46 & feminino & caso 4 dedo 2 & & \\
\hline 10 & caso 5 dedo 2 & & & caso 4 dedo 3 & & \\
\hline 11 & caso 6 dedo 1 & 53 & masculino & caso 5 dedo 1 & 47 & masculino \\
\hline 12 & caso 6 dedo 2 & & & caso 5 dedo 2 & & \\
\hline 13 & caso 7 dedo 1 & 21 & masculino & caso 5 dedo 3 & & \\
\hline 14 & caso 7 dedo 2 & & & caso 6 dedo 1 & 33 & masculino \\
\hline 15 & caso 8 dedo 1 & 41 & masculino & caso 7 dedo 1 & 29 & masculino \\
\hline 16 & caso 9 dedo 1 & 27 & feminino & caso 8 dedo 1 & 22 & feminino \\
\hline 17 & caso 10 dedo 1 & 31 & masculino & caso 9 dedo 1 & 33 & masculino \\
\hline 18 & caso 10 dedo 2 & & & caso 10 dedo 1 & 29 & masculino \\
\hline 19 & caso 11 dedo 1 & 39 & masculino & caso 10 dedo 2 & & \\
\hline \multirow[t]{3}{*}{20} & caso 12 dedo 1 & 29 & masculino & & & \\
\hline & Média & 35,00 & & Média & 33,00 & \\
\hline & Desvio padrão & 9,72 & & Desvio padrão & 10,42 & \\
\hline
\end{tabular}

palma da mão, na topografia dos nervos mediano, ulnar, radial e seus ramos, associado à sedação com propofol ou midazolam. No grupo 2 o paciente foi submetido à anestesia geral ou bloqueios anestésicos proximais (axilar ou interescalênico) também associados à sedação. No grupo 1, após a liberação cirúrgica das aderências, os pacientes foram despertados pelo anestesista utilizando 0,2 a $0,6 \mathrm{mg}$ de flumazenil e, a seguir, solicitados a realizar movimentos ativos de flexão e extensão. Em caso de não obtenção da movimentação desejada, a tenólise era então estendida até que a movimentação ativa atingisse a amplitude de movimentação passiva do dedo.

No grupo 2, após a liberação cirúrgica das aderências, os segmentos proximais dos tendões eram tracionados até a obtenção da amplitude de movimentação passiva do dedo (Figura 1).

Após a tenólise realizamos hemostasia criteriosa, fechamento da ferida operatória, curativos e enfaixamento da mão e dedos. No grupo 1, o paciente, ainda sob anestesia local, era estimulado a movimentar ativamente o dedo e observar o resultado conseguido em termos de ganho de amplitude de movimento (Figura 2). 


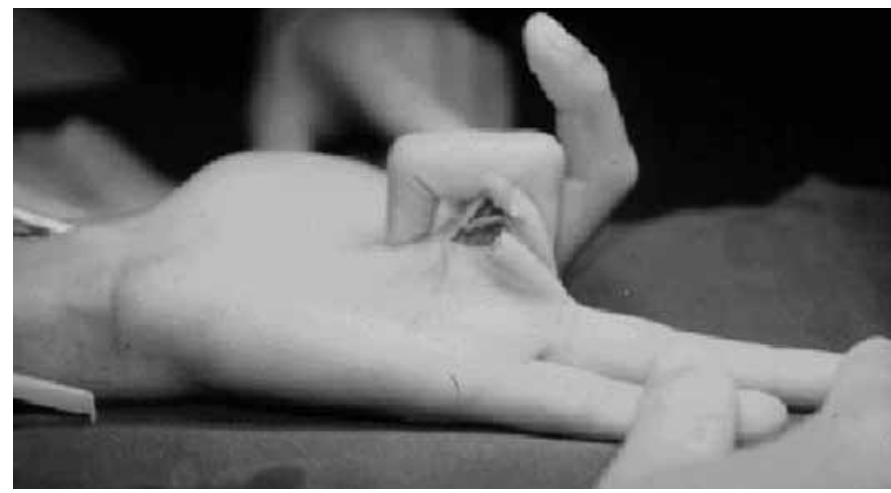

Figura 1 - Caso 2 do grupo 2 (tenólise sob anestesia geral). Notar a flexão do dedo conseguida graças à tração do tendão proximal na região do punho

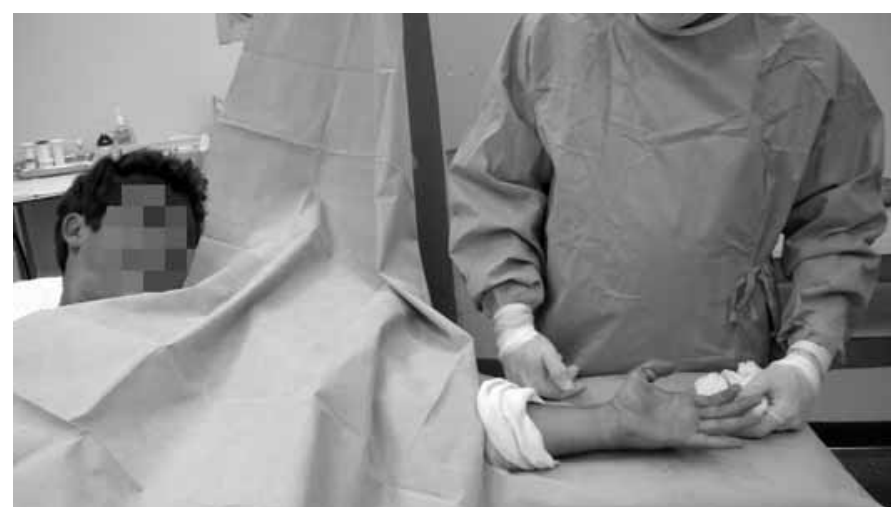

Figura 2 - Caso 8 do grupo 1 (tenólise sob anestesia locorregional com bloqueio dos nervos digitais do mediano). Notar que, após a liberação das aderências, o paciente consegue realizar a flexão e extensão ativa total do dedo comprometido

Os pacientes foram encaminhados a serviço de terapia da mão e seguiram o mesmo protocolo, que incluía movimentação passiva e ativa do dedo operado, combate ao edema e aderências tendinosas com enfaixamentos e massagem cicatricial.

Foram obtidas medidas da amplitude de movimentação das articulações interfalangianas proximal e distal antes da cirurgia e com seis meses de pós-operatório. As medidas do TAMs (total active motion modificado por Strickland) foram anotados e os valores dos dois grupos foram comparados estatísticamente pelos testes de Wilcoxon e Mann-Whitney. Foram adotados testes não paramétricos em virtude de as amostras não terem demosntrado distribuição normal.

\section{RESULTADOS}

Os valores de TAMs medidos no perído pré-operatório e com seis meses de pós-operatório no grupo 1 encontram-se na Tabela 2. A avaliação dos dados e o estudo estatístico demonstram que há uma diferença significativa entre os TAMs no pré e pós-operatório.
Tabela 2 - Distribuição das medidas do TAMs no grupo 1 no período pré-operatório (TAMs pré) e com seis meses de pósoperatório (TAMs pós)

\begin{tabular}{|c|c|c|c|}
\hline \multirow[t]{2}{*}{ Dedo } & \multicolumn{3}{|c|}{ Grupo 1} \\
\hline & & Valor de TAMs pré & Valor de TAMs pós \\
\hline 1 & caso 1 dedo 1 & 70,00 & 170 \\
\hline 2 & caso 1 dedo 2 & 65,00 & 165 \\
\hline 3 & caso 2 dedo 1 & 110,00 & 180 \\
\hline 4 & caso 2 dedo 2 & 90,00 & 185 \\
\hline 5 & caso 3 dedo 1 & 60,00 & 170 \\
\hline 6 & caso 4 dedo 1 & 65,00 & 165 \\
\hline 7 & caso 4 dedo 2 & 50,00 & 170 \\
\hline 8 & caso 4 dedo 3 & 110,00 & 180 \\
\hline 9 & caso 5 dedo 1 & 100,00 & 175 \\
\hline 10 & caso 5 dedo 2 & 70,00 & 180 \\
\hline 11 & caso 6 dedo 1 & 40,00 & 150 \\
\hline 12 & caso 6 dedo 2 & 50,00 & 140 \\
\hline 13 & caso 7 dedo 1 & 65,00 & 170 \\
\hline 14 & caso 7 dedo 2 & 55,00 & 180 \\
\hline 15 & caso 8 dedo 1 & 90,00 & 170 \\
\hline 16 & caso 9 dedo 1 & 70,00 & 180 \\
\hline 17 & caso 10 dedo 1 & 65,00 & 185 \\
\hline 18 & caso 10 dedo 2 & 60,00 & 180 \\
\hline 19 & caso 11 dedo 1 & 50,00 & 180 \\
\hline \multirow[t]{4}{*}{20} & caso 12 dedo 1 & 45,00 & 185 \\
\hline & Média & 69,00 & 173,00 \\
\hline & Desvio padrão & 20,69 & 11,63 \\
\hline & \multicolumn{3}{|c|}{ Teste de Wilcoxon $p<0,05^{*}$} \\
\hline
\end{tabular}

Os valores de TAMs medidos no perído pré-operatório e com seis meses de pós-operatório no grupo 2 encontram-se na Tabela 3. A avaliação dos dados demonstra que há uma diferença, comprovada por estudos estatísticos, entre os TAMs no pré e pós operatório.

A comparação das medidas de TAMs no período pré-operatório dos grupos 1 e 2 demonstra que estes são homogêneos, não havendo diferença estatística entre os dados obtidos (teste de Mann-Whitney $-\mathrm{p}>0,05)$.

A comparação das medidas de TAMs no período pós-operatório dos grupos 1 e 2 demonstra que eles apresentam resultados diferentes, havendo diferença estatisticamente significante entre os dados obtidos; demonstra que o grupo tratado com a técnica de despertar intraoperatório e anestesia locorregional (grupo 1) apresenta resultados superiores aos seis meses de pós-operatório (teste de Mann-Whitney - $\mathrm{p}<0,05$ ). 
Tabela 3 - Distribuição das medidas do TAMs no grupo 2 no período pré-operatório (TAMs pré) e com seis meses de pósoperatório (TAMs pós)

\begin{tabular}{|c|c|c|c|}
\hline \multirow[t]{2}{*}{ Dedo } & \multicolumn{3}{|c|}{ Grupo 2} \\
\hline & & Valor de TAMs pré & Valor de TAMs pós \\
\hline 1 & caso 1 dedo 1 & 80,00 & 150 \\
\hline 2 & caso 1 dedo 2 & 75,00 & 140 \\
\hline 3 & caso 1 dedo 3 & 90,00 & 130 \\
\hline 4 & caso 2 dedo 1 & 100,00 & 180 \\
\hline 5 & caso 3 dedo 1 & 70,00 & 160 \\
\hline 6 & caso 3 dedo 2 & 70,00 & 150 \\
\hline 7 & caso 3 dedo 3 & 90,00 & 130 \\
\hline 8 & caso 4 dedo 1 & 120,00 & 150 \\
\hline 9 & caso 4 dedo 2 & 110,00 & 140 \\
\hline 10 & caso 4 dedo 3 & 100,00 & 120 \\
\hline 11 & caso 5 dedo 1 & 60,00 & 150 \\
\hline 12 & caso 5 dedo 2 & 60,00 & 145 \\
\hline 13 & caso 5 dedo 3 & 70,00 & 140 \\
\hline 14 & caso 6 dedo 1 & 50,00 & 130 \\
\hline 15 & caso 7 dedo 1 & 60,00 & 180 \\
\hline 16 & caso 8 dedo 1 & 45,00 & 135 \\
\hline 17 & caso 9 dedo 1 & 50,00 & 140 \\
\hline 18 & caso 10 dedo 1 & 50,00 & 180 \\
\hline \multirow[t]{4}{*}{19} & caso 10 dedo 2 & 40,00 & 180 \\
\hline & Média & 73,16 & 148,95 \\
\hline & Desvio padrão & 23,17 & 18,90 \\
\hline & \multicolumn{3}{|c|}{ Teste de Wilcoxon $p<0,05^{\star}$} \\
\hline
\end{tabular}

\section{DISCUSSÃO}

O procedimento de tenólise dos flexores pode ser considerado um desafio para atingir o objetivo de melhorar a função digital. Há consenso em considerar que os pré-requistos para o sucesso incluem equipe ciúrgica experiente, paciente bem informado e motivado, e um programa cuidadoso de terapia da mão ${ }^{(9,14,15)}$. Em algumas situações não é possível determinar com exatidão o local e extensão das aderências tendinosas ${ }^{(9)}$. Para alguns cirurgiões, o uso da anestesia local oferece grande vantagem por proporcionar análise do tratamento de aderências tendinosas e liberações capsulares durante o ato cirúrgico ${ }^{(9,18)}$. Porém, não existem estudos que comprovem as vantagens do uso da anestesia locorregional e testes com despertar intraoperatório. As indicações para a tenólise abrangem situações clínicas em que a mobilidade passiva é bem maior que a ativa ${ }^{(13)}$. É também consenso que o sistema de polias deve ser preservado ou reconstruído nos procedimentos de tenólise, e que a movimentação ativa no intraoperatório pode facilitar esse procedimento ${ }^{(8,13,15)}$. Conduzindo adequadamente o tratamento, o procedimento de tenólise proporciona alto grau de satisfação do paciente ${ }^{(12,14,15)}$. Por outro lado, o procedimento de tenólise pode ser até mais difícil que a sutura primária, já que aborda uma área previamente operada e, em alguns pacientes, os resultados podem ser desastrosos, havendo piora da função, principalmente em casos de ruptura do tendão ${ }^{(17)}$.

Com o objetivo de avaliar o uso de procedimentos anestésicos locorregionais, decidimos comparar os resultados das tenólises realizadas por esse método com os obtidos com as tenólises convencionais com anestesia geral ou bloqueio total do plexo braquial. Nossa hipótese inicial é a de que pacientes submetidos à tenólise de flexores na zona 2 sob anestesia locorregional e ao despertar intraoperatório apresentam resultados mais satisfatórios, em virtude da melhor interpretação da liberação das aderências e preservação do sistema de polias proporcionadas pela movimentação ativa do tendão. Da mesma forma, o maior envolvimento do paciente no pósoperatório, gerado pela motivação em observar a recuperação da amplitude de movimento no intraoperatório, deve colaborar na obtenção de melhores resultados.

Com o objetivo de avaliar grupos homogêneos de pacientes, incluímos apenas os portadores de aderências tendinosas na zona 2, com cirurgias para sutura primária de tendões flexores entre três e 12 meses da indicação da tenólise, sem lesões associadas (fraturas, lesões ligamentares, perda do revestimento cutâneo, etc.) e sem outras patologias (diabetes, vasculopatias, artrose, artrite, etc.).

Os pacientes com critérios de inclusão foram então divididos nos grupos 1 (tenólise com anestesia locorregional e despertar intraoperatório) ou 2 (controle - tenólise com anestesia geral ou bloqueio total do plexo braquial).

A média de idade dos dois grupos foi parecida, sendo 35 anos para o grupo 1 e 33 para o grupo 2, não havendo também grande distinção significativa em relação ao sexo ( $75 \%$ dos pacientes do grupo 1 e $80 \%$ do grupo 2 eram do gênero masculino) (Tabela 1).

A técnica cirúrgica nos dois grupos também foi semelhante em relaçào à via de acesso, preservação do sistema de polias, preservação dos tendões flexores, liberação das aderências, hemostasia, fechamento da ferida, reabilitação e mobilização do dedo. A diferença entre os grupos baseou-se apenas na técnica de avaliação intraoperatória da tenólise; no grupo 1, os pacientes eram submetidos ao teste da flexão ativa do dedo após o despertar intraoperatório e, no grupo 2, os tendões eram tracionados pelo cirurgião em região proximal à tenólise. 
Todos os pacientes seguiram o mesmo protocolo de tratamento na reabilitação, incluindo exercícios ativos e passivos, combate ao edema, combate às aderências e treino de função. Os pacientes foram avaliados de acordo com a amplitude de movimentação articular medida pelo método de Strickland, que se baseia na soma da amplitude de flexão das articulações interfalangianas proximal e distal subtraída da deficiência de extensão destas mesmas articulações (TAMs - Total active motion modified by Strickland") ${ }^{(13)}$, no pré-operatório e aos seis meses de pós-operatório.

A análise dos dados obtidos no grupo 1 demonstra que a tenólise com anestesia locorregional e despertar intraoperatório proporciona bons resultados. Observamos grande melhora na amplitude de movimentação articular entre o período pré-operatório e aos seis meses da tenólise. Essa avaliação demonstra que a técnica adotada promove melhora da movimentação do dedo comprometido (Tabela 2). Da mesma forma, a análise dos dados obtidos no grupo 2 também demonstra que a tenólise convencional dos flexores sob anestesia geral ou bloqueio do plexo braquial proporciona bons resultados, promovendo melhora da movimentação (Tabela 3).

Quando comparamos os dados relativos à mobilidade ativa dos dedos comprometidos no perído pré-operatório (TAMs pré-operatório) dos grupo 1 e 2, observamos que não há diferenças em relação ao comprometimento. Tais dados também confirmam a homogeneidade das amostras, que foi um dos objetivos metodológicos deste estudo (Tabelas 2 e 3 ).

Ao compararmos os resultados da movimentação ativa total após seis meses da tenólise, observamos que o grupo de pacientes tratados com anestesia locorre- gional e despertar intraoperatório apresentava melhores resultados. Apesar de a tenolise de flexores na zona 2 realizada de forma convencional, utilizando anestesia geral ou bloqueio total do plexo braquial, proporcionar melhora da amplitude de movimentação dos dedos comprometidos, esta pode ser ainda implementada quando se utiliza anestesia locorregional e movimentação ativa com despertar intraoperatório. Talvez a movimentação ativa do dedo durante o ato cirúrgico, possível graças ao despertar intraoperatório e anestesia locorregional, possa ajudar a equipe cirúrgica a interpretar melhor se todas as aderências tendinosas foram realmente liberadas. Da mesma forma, a movimentação ativa do dedo pode ajudar a equipe cirúrgica a preservar estruturas nobres como o sistema de polias e estrutura tendinosa. Quanto ao paciente, parece que a lembrança da obtenção da movimentação ativa, durante o ato cirúrgico, é fator de estímulo e maior dedicação na reabilitação. Os pacientes submetidos à anestesia locorregional e despertar intraoperatório parecem ter o objetivo de, no mínino, manter o que foi conquistado durante o procedimento cirúrgico.

\section{CONCLUSÕES}

Este estudo prospectivo, comparativo e controlado permitiu concluir que a tenólise dos flexores na zona 2 , para tratamento das aderências tendinosas pós-suturas tendinosas primárias, proporciona bons resultados em termos de ganho de amplitude articular. Concluímos também que a tenólise sob anestesia locorregional com despertar intraoperatório permitindo a movimentação ativa do dedo proporciona resultados mais satisfatórios que a técnica convencional realizada com anestesia geral ou bloqueio total do plexo braquial.

\section{REFERÊNCIAS}

1. Kleinert HE, Kutz JE, Ashbell TS. Primary repair of lacerated flexor tendons in "no man's land". J Bone Joint Surg 1967;49:577.

2. Potenza AD. Tendon healing within the flexor digital sheath in the dog. J Bone Joint Surg Am. 1962;44(1):49-64.

3. Potenza AD, Herte MC. The synovial cavity as a "tissue culture in situ" - science or nonsense? J Hand Surg Am. 1982;7(2):196-9.

4. Manske PR, Lesker PA. Biochemical evidence of flexor tendon participation in the repair process - an in vitro study. J Hand Surg Br. 1984;9(2):117-20.

5. Manske PR, Gelberman RH, Vande Berg JS, Lesker PA. Intrinsic flexor-tendon repair. A morphological study in vitro. J Bone Joint Surg Am. 1984;66(3):385-96.

6. Becker H, Graham MF, Cohen IK, Diegelmann RF. Intrinsic tendon cell proliferation in tissue culture. J Hand Surg Am. 1981;6(6):616-9

7. Manske PR, Gelberman RH, Lesker PA. Flexor tendon healing. Hand Clin. 1985;1(1):25-34.

8. Strickland JW. Management of flexor tendon injuries. Orthop Clin North Am. 1983;14(4):827-46.

9. Schneider LH. Tenolysis and capsulectomy after hand fractures. Clin Orthop Relat Res. 1996;(327):72-8.

10. Whitaker JH, Strickland JW, Ellis RK. The role of flexor tenolysis in the palm and digits. J Hand Surg Am. 1977;2(6):462-70.

11. Ketchum LD. Primary tendon healing: a review. J Hand Surg Am. 1977;2(6):428-35.

12. Strickland JW. Flexor tendon injuries. Part 5. Flexor tenolysis, rehabilitation and results. Orthop Rev. 1987;16(3):137-53.

13. Strickland JW. Flexor tenolysis. Hand Clin. 1985;1(1):121-32.

14. Eggli S, Dietsche A, Eggli S, Vögelin E. Tenolysis after combined digital injuries in zone II. Ann Plast Surg. 2005;55(3):266-71.

15. Azari KK, Meals RA. Flexor tenolysis. Hand Clin. 2005;21(2):211-7

16. Feldscher SB, Schneider LH. Flexor tenolysis. Hand Surg. 2002;7(1):61-74.

17. Goloborod'ko SA. Postoperative management of flexor tenolysis. J Hand Ther. 1999;12(4):330-2.

18. Hunter JM, Schneider LH, Dumont J, Erickson JC 3rd. A dynamic approach to problems of hand function using local anesthesia supplemented by intravenous fentanyl-droperidol. Clin Orthop Relat Res. 1974;(104):112-5. 